\begin{tabular}{|c|l|}
\hline Title & Electron scattering due to confined and extended acoustic phonons in a quantum wire \\
\hline Author(s) & Nishiguchi, Norihiko \\
\hline Citation & $\begin{array}{l}\text { Physical Review B, 54(3), 1494 1497 } \\
\text { https://doi.org/10.1103/PhysRevB.54.1494 }\end{array}$ \\
\hline Issue Date & 1996-07-15 \\
\hline Doc URL & http://hdl.handle.net/2115/47088 \\
\hline Rights & ○ 1996 The A merican Physical Society \\
\hline Type & article \\
\hline File Information & PhysRevB.54.1494.pdf \\
\hline
\end{tabular}

Instructions for use 


\title{
Electron scattering due to confined and extended acoustic phonons in a quantum wire
}

\author{
Norihiko Nishiguchi \\ Department of Applied Physics, Hokkaido University, Sapporo 060, Japan
}

(Received 22 March 1996)

\begin{abstract}
Electron scattering due to confined and extended acoustic phonons is analytically investigated for a quasione-dimensional GaAs quantum wire embedded within bulk AlAs. It is found that the deformation coupling between electrons and confined phonons is extremely small in comparison with that between electrons and extended phonons. Consequently, the electron scattering processes are governed by the extended phonons, and the magnitude and electron energy dependence of the scattering rates are almost the same as those due to the usual three-dimensional bulk phonons. [S0163-1829(96)08627-4]
\end{abstract}

Recent analytical studies on acoustic-phonon modes of a quasi-one-dimensional (Q1D) wire within another bulk material have revealed that the embedded Q1D wires support confined and interface acoustic-phonon modes, ${ }^{1,2}$ as well as extended phonon modes. The extended phonon modes are also modulated by the Q1D wire structures, and resonant modes related to the wire dimensions appear. ${ }^{3}$ These results show that the acoustic-phonon modes of the embedded Q1D wires are different from the usual 3D bulk phonon modes. However, the modifications in acoustic-phonon modes were overlooked and the usual 3D bulk phonon modes were assumed for the studies on electron transport phenomena of the Q1D wires. ${ }^{4-8}$

The effects of confined acoustic phonons on electron transport in a Q1D free-standing wire have been theoretically studied, and the scattering rates are found to substantially increase, due to confined phonon emission. ${ }^{9,10}$ In addition, conductance fluctuations, due to confined acoustic phonons, were experimentally observed in metal wires deposited on an insulating substrate, ${ }^{11}$ where extended and confined phonons coexist. Hence, we may expect modifications in electron transport phenomena of the embedded Q1D wires by the confined acoustic phonons, similar to the increase of scattering rates in Q1D free-standing wires, ${ }^{9,10}$ while optical phonons confined in embedded quantum well structures slightly affect electron transport. ${ }^{12-14}$ In this paper, we make clear the modifications in electron scattering of the embedded Q1D wires, due to the emission and absorption of both confined and extended acoustic phonons, by examining the electron scattering rates within the lowest-energy subband via the deformation potential. In this work, we model the Q1D wire to be a cylinder, where acoustic-phonon modes, as well as electron wave functions, can be rigorously derived.

Considering electrons confined in a cylindrical GaAs quantum wire $(A)$ with radius $R$ within bulk AlAs $(B)$, where the wire axis is taken as the $z$ direction, the electron energy of the lowest subband is given within the effectivemass and parabolic-band approximations by

$$
E_{k_{z}}=\frac{\hbar^{2} k_{z}^{2}}{2 m^{*}},
$$

where $k_{z}$ is the longitudinal wave number and $m^{*}$ denotes the effective mass. The corresponding electron wave func- tion $\left|k_{z}\right\rangle$ is given, within the infinite potential well model, ${ }^{15}$ by

$$
\left\langle\mathbf{r} \mid k_{z}\right\rangle=\frac{1}{\sqrt{\pi R^{2} L_{w}}} \frac{J_{0}\left(k_{\rho} \rho\right)}{J_{1}\left(k_{\rho} R\right)} e^{i k_{z} z}
$$

in the cylindrical coordinates $(\rho, \varphi, z)$. Here, $J_{0(1)}(x)$ denotes the first kind of the Bessel function of the order zero (unity) and $L_{w}$ is the wire length. $k_{\rho}$ is the lateral wave number given by the smallest root of $J_{0}\left(k_{\rho} R\right)=0$.

Since the translational symmetry in the lateral direction of the system is lost due to the wire structure, neither longitudinal (LA) nor transverse acoustic (TA) waves is a normal phonon mode. These acoustic waves are coupled at the wiresurrounding interface, being a normal mode. Assuming isotropic elasticity of the constituent materials, phonon modes have certain rotational symmetries about the wire axis owing to the full axial symmetry of the system, and they are classified into two azimuthally symmetric modes referred to as the dilatational and torsional modes and azimuthally varying modes termed flexural modes. ${ }^{16}$ The total rotational symmetry about the wire axis of electron wave functions and acoustic-phonon modes has to be conserved for emission and absorption of acoustic phonons via the deformation potential. Because the rotational symmetry of electron wave functions remains unchanged for intrasubband transitions and because the torsional mode does not induce volume change, only the dilatational mode is responsible for intrasubband transitions.

The displacement vector field $\mathbf{u}$ of the dilatational mode is expressed in both the wire and surrounding medium in terms of a scalar potential $\phi_{A(B)}$ and a vector potential $\boldsymbol{\Psi}_{A(B)}$ as

$$
\mathbf{u}_{\alpha}(\mathbf{r})=\chi_{\alpha \ell} \nabla \phi_{\alpha}(\mathbf{r})+\chi_{\alpha t} \nabla \times \nabla \times \boldsymbol{\Psi}_{\alpha}(\mathbf{r}),
$$

for $\alpha=A$ and $B$. The vector potential $\Psi_{\alpha}(\mathbf{r})$ is given by $\mathbf{e}_{z} \psi_{\alpha}(\mathbf{r})$, where $\mathbf{e}_{z}$ is the unit vector along the wire axis. The potential functions $\phi_{\alpha}$ and $\psi_{\alpha}$ obey wave equations of scalar fields with sound velocities of LA waves $v_{\alpha \ell}$ and TA waves $v_{\alpha t}$, respectively, given by the Bessel and modified Bessel functions of order zero. Applying the boundary condition of continuity of displacement and stress fields at the wiresurrounding interface, we obtain confined and extended modes, ${ }^{1-3}$ as the normal phonon modes of this system. Interface phonon modes do not exist for the combination of 


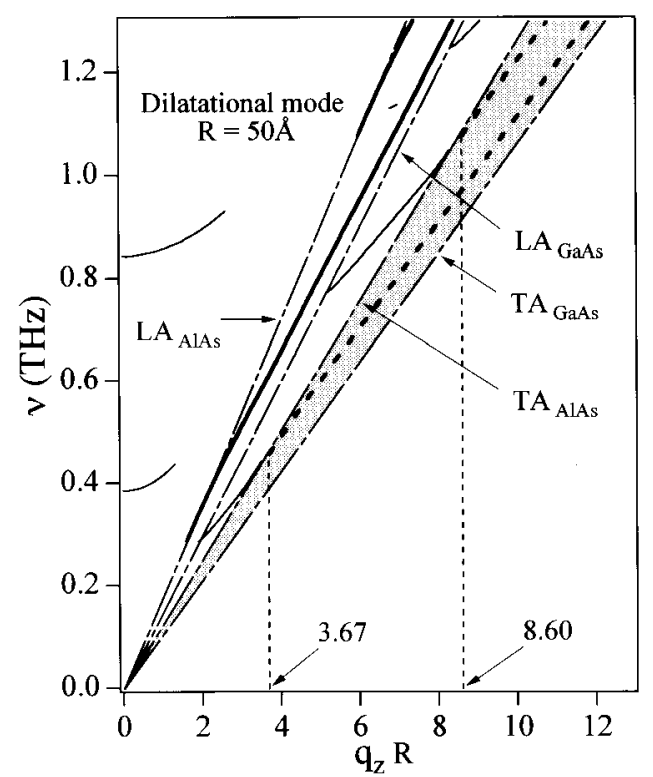

FIG. 1. Dispersion relations of confined and resonant acousticphonon modes. The dashed lines are the dispersion curves of the confined dilatational phonon mode. The thin and thick solid lines denote the dispersion curves of resonant modes. The dot-dashed lines are the dispersion curves of bulk LA and TA waves of GaAs and AlAs. The dispersion curves of confined modes begin with the cutoff longitudinal wave numbers, at which the dispersion curves intersect the bulk TA waves of AlAs.

GaAs/AlAs. ${ }^{2}$ The coefficients $\chi$ are determined by the boundary condition and the following normalization condition:

$$
\int_{V} \varrho(\mathbf{r}) \mathbf{u}_{q_{z} \omega}^{L} *(\mathbf{r}) \cdot \mathbf{u}_{q_{z} \omega}^{L}(\mathbf{r}) d \mathbf{r}=\frac{\hbar}{2 \omega_{L}}
$$

where the mass density $\varrho(\mathbf{r})$ takes a value $\varrho_{A}$ in the wire or $\varrho_{B}$ in the surrounding medium. Here, $q_{z}$ and $\omega$ are the longitudinal wave number and an angular frequency of phonons, respectively. $L$ denotes the confined and extended phonon modes. Figure 1 shows the dispersion relations of the confined dilatational mode (dashed lines). They are limited to the frequency region between the dispersion curves of the bulk TA waves of wire and surrounding materials, and each dispersion curve of the confined mode begins with the finite cutoff longitudinal wave numbers $q_{c}$. The reduced cutoff longitudinal wave number $q_{c} R$ is 3.67 for the lowest phonon subband and 8.60 for the second subband. Confined phonon modes with $q_{z}$ smaller than $q_{c}(=3.67 / R)$ do not exist.

Extended phonon modes have a large amplitude in the wire region at certain frequencies $\omega$ and longitudinal wave numbers $q_{z}$. We refer to the extended modes, with such large amplitude in the wire region, as resonant modes $^{3}$ and term the trajectories of the frequencies and longitudinal wave numbers in the $\nu-q_{z}$ plane the dispersion relations of the resonant modes, which are also illustrated in Fig. 1 by thin and thick solid lines. It should be noted here that the amplitude in the wire region on the thick dispersion curves becomes an order of magnitude larger than that in the vicinity of the dispersion curves, leading to a substantial increase of the electron-extended phonon coupling.
The interaction Hamiltonian $H_{e-\mathrm{ph}}$ is given, in terms of annihilation and creation operators of phonons $a_{q_{z} \omega_{L}}^{L}$ and $a_{q_{z} \omega_{L}}^{L^{\dagger}}$, by

$$
\begin{aligned}
H_{e-\mathrm{ph}}= & -\Xi \sum_{L} \sum_{q_{z}, \omega_{L}}\left(\frac{\omega_{L}}{v_{A, \ell}}\right)^{2}\left[a_{q_{z} \omega_{L}}^{L} \chi_{A, C} \phi_{A, q_{z} \omega}^{L}(\mathbf{r})\right. \\
& \left.+a_{q_{z} \omega_{L}}^{L \dagger} \chi_{A, \ell}^{*} \phi_{A, q_{z} \omega}^{L *}(\mathbf{r})\right]
\end{aligned}
$$

where $\Xi$ is the deformation potential, which is set to be $7 \mathrm{eV}$ in this paper.

The scattering rates of an electron with $k_{z}$, due to acoustic phonon emission $(+)$ and absorption $(-)$, are calculated from Fermi's golden rule as

$$
\begin{aligned}
w^{( \pm)}= & \frac{8 \pi \Xi^{2}}{\hbar v_{A, \ell}{ }^{4}} \sum_{L} \sum_{k_{z}^{\prime}, q_{z}, \omega_{L}}\left|\chi_{A, \ell}\right|^{2} \omega_{L}^{4}\left[N_{\omega_{L}}+\frac{1}{2} \pm \frac{1}{2}\right] F\left(q_{A, \rho}\right)^{2} \\
& \times \delta_{k_{z^{\prime}}, k_{z} \mp q_{z}} \delta\left(E_{k_{z}^{\prime}}-E_{k_{z}} \pm \hbar \omega_{L}\right) .
\end{aligned}
$$

$N_{\omega_{L}}$ is the Bose-Einstein distribution function for phonons. The radial wave number $q_{A, \rho}$ of acoustic phonons is defined by $q_{A, \rho}=\sqrt{\left|\omega^{2}-v_{A, C}^{2} q_{z}^{2}\right|} \cdot F(x)$ is the overlap integral of the electron wave function and the potential function of phonons, and becomes

$$
F\left(q_{A, \rho}\right)=\frac{1}{R^{2} J_{1}\left(k_{\rho} R\right)^{2}} \int_{0}^{R} J_{0}\left(k_{\rho} \rho\right)^{2} I_{0}\left(q_{A, \rho} \rho\right) \rho d \rho,
$$

for $\omega<v_{A, \ell} q_{z}$ and

$$
F\left(q_{A, \rho}\right)=\frac{1}{R^{2} J_{1}\left(k_{\rho} R\right)^{2}} \int_{0}^{R} J_{0}\left(k_{\rho} \rho\right)^{2} J_{0}\left(q_{A, \rho} \rho\right) \rho d \rho,
$$

for $v_{A, \ell} q_{z}<\omega$.

The scattering rates, due to the usual 3D bulk phonons, can be formulated straightforwardly, by means of the same approximation for electron wave functions (2), as

$$
\begin{aligned}
w_{3 \mathrm{D}}^{( \pm)}= & \frac{4 \pi \Xi^{2}}{\rho_{A} v_{A, \ell^{2}}} \frac{1}{V} \sum_{k_{z}^{\prime}, \mathbf{q}} \omega_{\mathbf{q}}\left[N_{\omega_{\mathbf{q}}}+\frac{1}{2} \pm \frac{1}{2}\right] \\
& \times F\left(q_{\rho}\right)^{2} \delta_{k_{z^{\prime}, k_{z}} \mp q_{z}} \delta\left(E_{k_{z}^{\prime}}-E_{k_{z}} \pm \hbar \omega_{\mathbf{q}}\right),
\end{aligned}
$$

where $\omega_{\mathbf{q}}=v_{A, \ell}|\mathbf{q}|$.

Figure 2 shows the scattering rates, due to both confined and extended acoustic phonons versus electron energy for $R=50 \AA$ at temperature $77 \mathrm{~K}$, as well as those due to the usual 3D bulk phonons. The resultant scattering rates vary smoothly with respect to electron energy, and there is no peak of scattering rates. As for the magnitude and electron energy dependence of the scattering rates, there is not a noticeable difference between the results and the scattering rates owing to the usual 3D bulk phonons.

To examine these results, the scattering rates solely attributed to the confined phonons of the lowest phonon subband are plotted in Fig. 2. The electron scattering processes, due to emission or absorption of confined phonons, take place at above an electron energy $E_{0}=74.4 \mathrm{meV}$. This stems from the absence of confined phonons with small longitudinal wave numbers. A change of longitudinal wave numbers of 


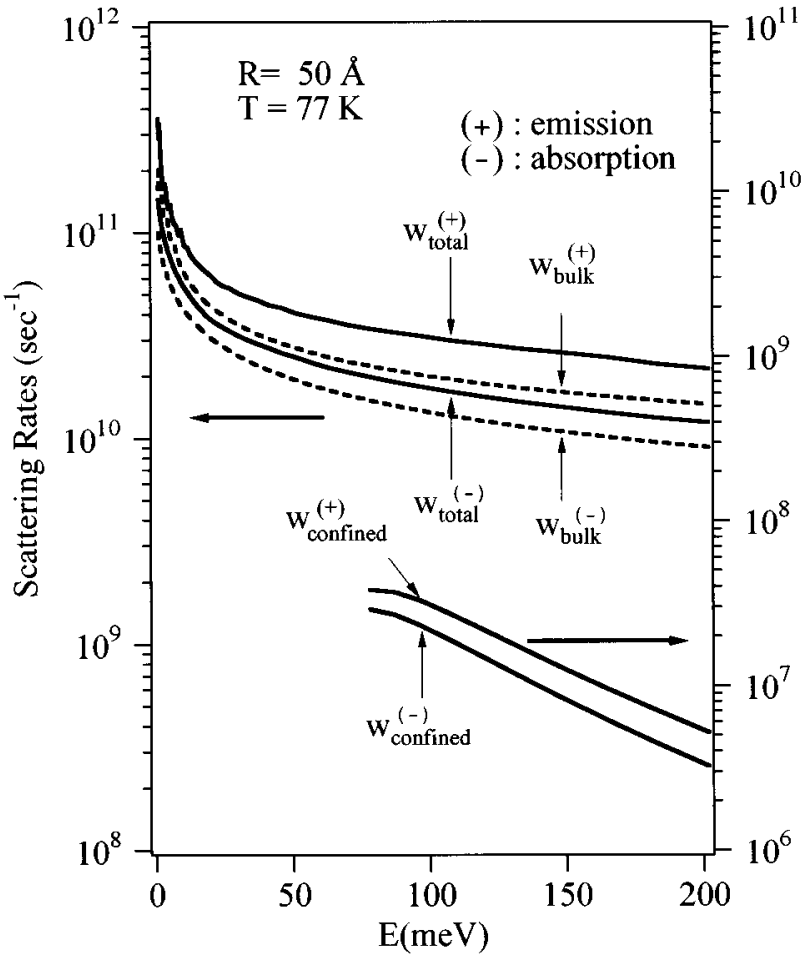

FIG. 2. The scattering rates $w_{\text {total }}^{( \pm)}$, due to confined and extended acoustic phonons of a cylindrical GaAs wire with $R=50 \AA$ within bulk AlAs versus electron energy, together with those $w_{\text {bulk }}^{( \pm)}$due to acoustic bulk phonons. The scattering rates $w_{\text {confined }}^{( \pm)}$solely due to confined phonons are also plotted.

electrons, due to emission or absorption of an acoustic phonon, which is approximately equal to $2 k_{z}$, is equivalent to the longitudinal wave number $q_{z}$ of the phonon, i.e., $2 k_{z} \approx q_{z}$. Because the longitudinal wave number $q_{z}$ of confined acoustic phonons is greater than the cutoff wave number $q_{c}$, the scattering processes owing to confined acoustic phonons should occur at above the critical electron energy $E_{\mathrm{cr}} \simeq\left(\hbar^{2} / 2 m^{*}\right)\left(q_{c} / 2\right)^{2}$. The critical electron energy $E_{\mathrm{cr}}$ for the lowest phonon subband yields $76.6 \mathrm{meV}$ for $R=50 \AA$, which almost agrees with $E_{0}$, and that for the second subband becomes $421 \mathrm{meV}$. Thus, the electron scattering processes associated with confined phonons take place above $E_{\mathrm{cr}}$. In contrast, there is no cutoff wave number for acoustic phonons of free-standing wires. Then electron scattering processes due to confined acoustic phonons in a free-standing wire occur whenever the momentum and energy conservation laws are satisfied.

Figure 2 also shows that the scattering rates only due to confined phonons are two orders of magnitude smaller than those due to both confined and extended phonons. The dilatational confined mode consists of the LA and TA waves, as described above. The LA wave component, which is important for the deformation potential scattering, varies with frequency and longitudinal wave number. ${ }^{3}$ Figure 3 illustrates the relative contribution of the LA wave to the dilatational confined mode. The absolute magnitude of the coefficient of LA wave, $\left|\chi_{A}\right|$, becomes $0.06\left|\chi_{A t}\right|$ at most, and decreases with increasing electron energy. From that fact, the confined phonon modes are found almost to be the TA waves, inducing little local volume change. The deformation coupling be-

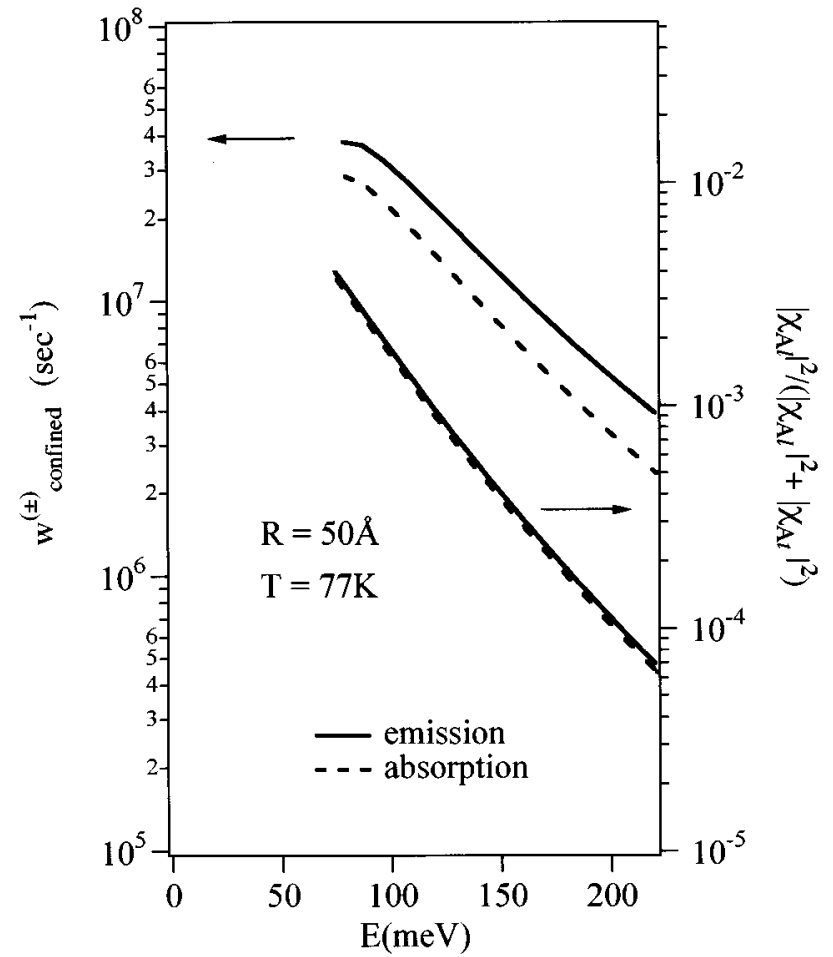

FIG. 3. The scattering rates due to confined acoustic phonons in a cylindrical GaAs wire with $R=50 \AA$ versus electron energy, and the corresponding relative magnitude of the coefficients, $\left|\chi_{A}\right|^{2} /$ $\left(\left|\chi_{A}\right|^{2}+\left.\chi_{A t}\right|^{2}\right)$.

tween electrons and confined acoustic phonons, therefore, becomes very small, and the electron scattering processes due to confined acoustic phonons, as a result, merely take place as compared with those due to extended phonons.

In contrast to the confined phonons, the extended phonons induce local volume change. In particular, the resonant modes of the thick dispersion curves are almost the LA waves, i.e., $\mid \chi_{A} \lambda^{2} /\left(\left|\chi_{A}\right|^{2}+\left|\chi_{A t}\right|^{2}\right) \approx 1$, and have a large amplitude in the wire region, ${ }^{3}$ as mentioned above. This leads to large electron-extended phonon coupling, then we may expect some modification in electron scattering rates, due to the resonant mode phonons. However, there is not conspicuous behavior of electron scattering rates because the effects of the resonant mode phonons on electron scattering are smeared by the integrated contribution of all extended phonons.

In conclusion, although the acoustic-phonon modes in the Q1D wire embedded within another bulk material are remarkably modified, the modifications in acoustic-phonon modes are not reflected in electron scattering rates. The resultant scattering rates almost coincide, in both magnitude and electron energy dependence, with the scattering rates assuming the usual acoustic bulk phonons. It is because the deformation coupling between electrons and confined phonons is very small, in comparison with the coupling with extended phonons. Since, as mentioned above, confined acoustic phonons cause the detectable modifications in electron transport in a free-standing wire and wires on a substrate, the weak coupling between confined acoustic phonons and electrons is peculiar to the embedded wires. The weak 
coupling stems from that the confined acoustic-phonon modes are effectively the TA waves, which is independent of the wire dimensions. Hence, the bulk acoustic-phonon ap- proximation is found to be valid for electron scattering in the embedded Q1D wires like electron scattering with optical phonons. $^{12-14}$
${ }^{1}$ N. Nishiguchi, Jpn. J. Appl. Phys. 33, 2852 (1994).

${ }^{2}$ N. Nishiguchi, Phys. Rev. B 50, 10970 (1994).

${ }^{3}$ N. Nishiguchi, Phys. Rev. B 52, 5279 (1995).

${ }^{4}$ V. K. Arora, Phys. Rev. 23, 5611 (1981).

${ }^{5}$ J. Lee and M. O. Vassell, J. Phys. C 17, 2525 (1984).

${ }^{6}$ G. Fishman, Phys. Rev. B 36, 7448 (1987).

${ }^{7}$ U. Bockelmann and G. Bastard, Phys. Rev. B 42, 8947 (1990).

${ }^{8}$ B. Tanatar, J. Phys. Condens. Matter 5, 2203 (1993).

${ }^{9}$ S. Yu, K. W. Kim, M. A. Stroscio, G. J. Iafrate, and A. Ballato, Phys. Rev. B 50, 1733 (1994).

${ }^{10}$ S. Yu, K. W. Kim, M. A. Stroscio, and G. J. Iafrate, Phys. Rev. B 51, 4695 (1995).
${ }^{11}$ J. Seyler and M. N. Wybourne, Phys. Rev. Lett. 69, 1427 (1992).

${ }^{12}$ T. Tsuchiya and T. Ando, Phys. Rev. B 47, 7240 (1993).

${ }^{13}$ H. Ruecker, E. Molinari, and P. Lugli, Phys. Rev. B 45, 6747 (1992).

${ }^{14}$ F. Rossi, L. Rota, C. Bungaro, P. Lugli, and E. Molinari, Phys. Rev. B 47, 1695 (1993).

${ }^{15}$ A finite potential well and the corresponding wave functions will give more quantitative results. On this subject, see N. N. Constantinou and B. K. Ridley, J. Phys. Condens. Matter 1, 2283 (1989).

${ }^{16}$ B. A. Auld, Acoustic Fields and Waves (Wiley, New York, 1973), Vol. II, Chap. 10. 